\title{
Organization Type Code
}

National Cancer Institute

\section{Source}

National Cancer Institute. Organization Type Code. NCI Thesaurus. Code C93878.

A coded value specifying the kind of organization. 